\title{
Research on Fiber-grating-based Wireless Sensor Networks
}

\author{
Bo LIU, Jinjie CHEN, Hao ZHANG, and Xiaoyi DONG
}

Key Laboratory of Photoelectric Information Technical Science, Ministry of Education,Institute of Modern Optics, Nankai University, Tianjin, 300071, China

*Corresponding author: Jinjie CHENＥ-mail: jasoncjj2007@163.com

\begin{abstract}
A 20-node fiber-grating-based wireless sensor network is proposed and experimentally demonstrated. Each sensor node is integrated with the light source, 1-3 FBG sensing probes, wavelength demodulation, and wireless communication module. Via self-organized clusters and low energy adaptive clustering hierarchy $(\mathrm{LEACH})$ route protocols, the sensor nodes are able to exchange sensing data with the control center, and the maximum communication radius of a sensor node is over $170 \mathrm{~m}$. The sensor node is battery-powered with a survival lifetime of up to 120 days at a network refresh rate of 5 minutes.
\end{abstract}

Keywords: Fiber sensor, wireless sensor networks, LEACH

\section{Introduction}

Multi-parameter, multi-function, and distributed real-time sensing networks have been of great interests in the past decades. Due to its good reliability, electro-magnetism immunity, simple structure, high sensitivity and longevity, the fiber-grating-based sensing technique has become a distinguished approach in the field of the sensing technology. Currently, the detection of various physical parameters, including temperature, strain, etc., has been realized with the fiber-optic sensing technology [1-13].

As the fiber sensing networks turn to be the inevitable trend in the development of the fiber sensing technology, a single sensor can not meet the demand of the sensing system any longer. Generally speaking, owing to the different central reflection wavelengths of different fiber sensors, it would be convenient to construct a small scale fiber Bragg grating (FBG) sensor array by adopting the wavelength division multiplexing (WDM), time division multiplexing (TDM), space division multiplexing (SDM), and mixed multiplexing (WDM+TDM+SDM) technologies. Researchers around the world have deeply studied the fiber grating multiplexing technology [14-19]. Youlong Yu et al. conducted a de-multiplexing experiment on a $3 \times 10$ fiber grating sensor array in 2002 [17]. Another work that should be noted was Pengchun Peng's experiment. In 2004, he and a few co-workers achieved parallel de-multiplexing for multi-channel sensing signals based on the optical intensity multiplexing and WDM techniques. However, in his scheme the fluctuation of the optical intensity across different sensing channels had an obvious influence on the demodulation resolution of the sensing system [15]. Additionally, in 2005 Libo Yuan presented a fiber sensor network topology based on two low coherent loops [19].

However, a shared light source is necessary for all of the above fiber grating sensing networks. Due to this disadvantage, in most cases the scale of the sensor array is limited to not more than tens of

Received: 12 September 2011 / Revised version: 27 December 2011

(C) The Author(s) 2012.This article is published with open access at Springerlink.com 
nodes by the transmission bandwidth of the light source and fiber connection losses. Moreover, as the fiber is required for the connection between different sensors, the extensionality and flexibility of the sensing networks are also limited to a large extend. Thus, the conventional fiber grating sensing networks are no longer able to meet the demand of the large scale, distributed, and extensional network technology.

Fortunately, wireless sensor networks have become the mainstream with the development of the modern sensing technology. Wireless sensor networks (WSNs) are self-organized by a great amount of sensor nodes based on the wireless communication technology. Quantification of the data acquisition, data fusion, and transmission application could be realized in WSNs. In these networks, many sensor nodes are deployed in the monitor area, and the self-organization is realized wirelessly to collaboratively sense, acquire, process the monitor information over the networks area, and send the results to the observers. As the information system is integrated with distributed information acquisition, transmission, and processing techniques, WSNs have attracted world-wide interest for their low cost, miniaturization, low power consumption, networking flexibility, ease for configuration, and capability of dealing with moving objects. Playing an important role in the development of the national economy and national security, the WSN technology is publicly considered as the top one amongst the ten key technologies that may potentially affect the future of the mankind.

Around the 1980s, the United States National Defense Senior Project Agency presented a distributed sensing network (DSN) scheme and after that accomplished the study of the sensor, communication, and data processing techniques, and the development of the distributed software [20]. Thereafter, a few researchers at Carnegie Maroon University (CMU) developed a DSN operation system named "Accent", which supported the transparent network, system configuration, and re-binding [21]. In the late 1990s, intelligent sensors were connected with the sensor controller via the scene BUS to constitute a local network. Since then, the sensor network technology, especially the wireless sensor network technology, has been extensively studied and developed. Currently, the studies on WSNs are focused on the following aspects: network construction, routing and data transfer, linking protocol, application of IPv6 in the WSN, data acquisition and capture technique, data processing technique, database technique [20-35], power consumption management, location technique, and the development of the software environment, software, simulator, and application system for the WSN, etc. In 2001, considering sensor network as an important research field, the United States National Defense Ministry presented the C4KISR plan that aimed to set up a series of research projects on the military sensor network. Intel Corporation put forward a development plan for the new generation computing technology based on micro-sensor networks in 2002. And in 2003, the American Natural Science Foundation (NSF) also started a research plan on sensor networks. Japan, Great Britain, Italy and Brazil became interested in the sensor network technology and began to conduct research in this field. In March 2006, on IEEE Internet Computing, Krishna Chintalapudi et al. published their work on building health monitors based on the WSNs system. And in the same issue, Geoffrey Werner-Allen published his work on the volcano monitor of Volcán Reventador based on the WSNs system [35].

In summary, we could draw a conclusion that although fiber grating sensors have many advantages, the scale and cost of networks are limited by conventional approaches. Owing to its flexible and convenient network configuration, good extensionality and flexibility, WSNs would inevitably become the mainstream technology with the development of large scale and super large scale sensor networks. Based on our previous work and by exploiting the merits of the fiber grating based 
wireless sensor network technology, we proposed and demonstrated a fiber-grating-based wireless sensor network for the application of the multi-parameter sensing measurement. Fiber gratings with the high sensitivity and immunity to external perturbations were employed in this system, and the low-cost fiber-grating-based wireless sensor module was successfully designed. Via the WSNs, different sensors could exchange information, collaborate and accomplish data extraction, data analysis and determination.

In our experiment, a 20-node fiber-grating-based wireless sensing network has been achieved for the demonstration. Based on the same method, the network capacity can be easily expanded. Our research results would be expected to pave a new way for the application of fiber grating sensors and provide new methods and approaches for the safety and health monitors under different complicated and severe circumstances, making fiber sensors with their original good features more flexible and convenient for the construction of large scale sensor networks.

\section{Structure of the fiber-grating-based WSNs}

Figure 1 shows the structural diagram of our proposed fiber-grating-based wireless sensor network, including the following parts:

(1) Sensor node: acquires fiber grating sensing signals, performs data fusion in the data layer, and transfers the data to sink nodes.

(2) Sink node: receives data from sensor nodes, performs the data fusion in the status layer, and transfers data to management nodes.

(3) Management node: performs the data fusion in the characteristics layer, and transfers data to the remote control center via the global system for mobile communication (GSM) or Internet to achieve the trans-region monitor.

(4) Central control and terminal: receives and displays measured physical parameters, and sends control signals.

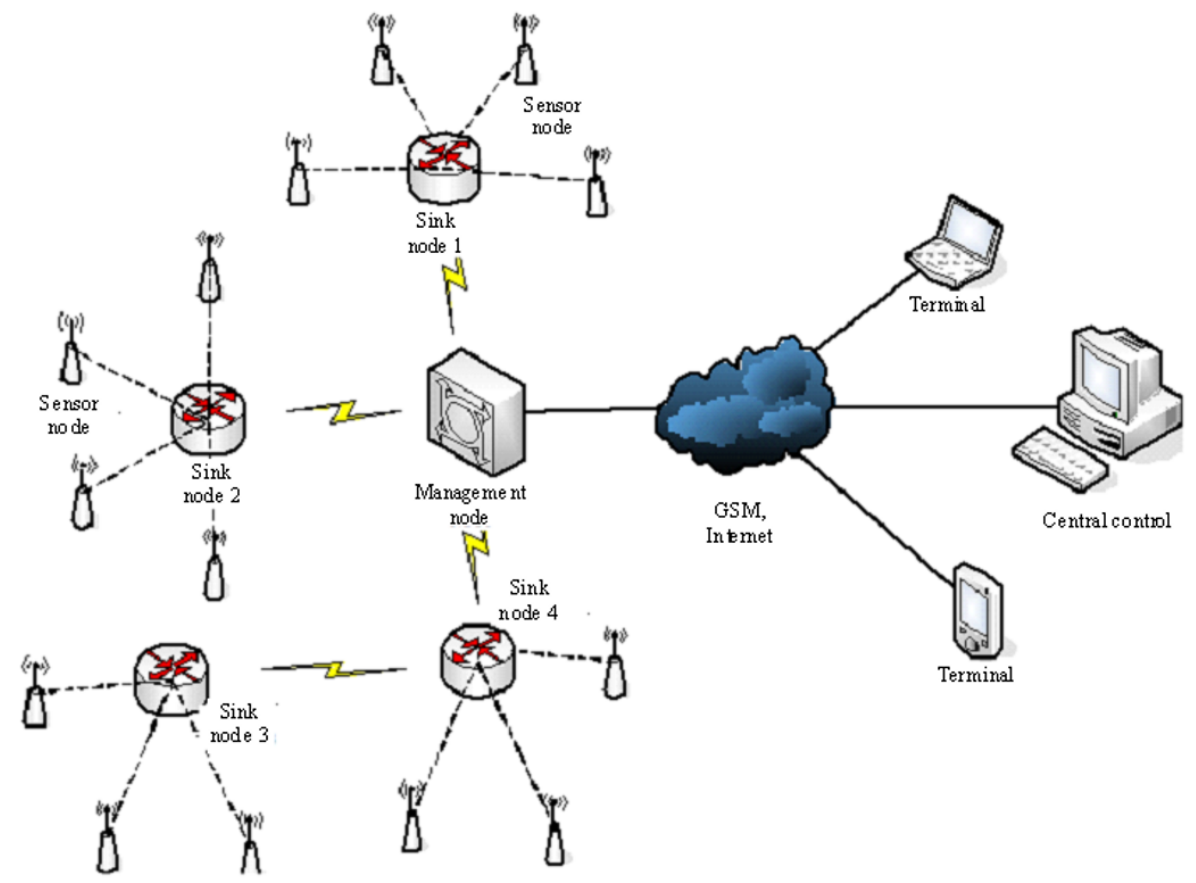

Fig. 1 Structural diagram of the proposed fiber-grating-based WSN.

\subsection{Sensor nodes}

Sensor nodes are the core units of the whole wireless network. Their basic function is to acquire and demodulate the fiber grating sensing information and wirelessly send the sensing outcome to other sensors and sink nodes to 
accomplish the data exchange. Functionally speaking, the conventional WDM fiber grating sensing system is integrated into the sensor nodes, and the wavelength demodulation and measurand calculation are performed via the digital signal processing (DSP). The block diagram of functional units is shown in Fig. 2. According to the characteristics of wireless sensor networks, our work could be categorized into the following 4 aspects:

(1) Design of the fiber grating multi-parameter sensing probe

Based on the sensing principle of fiber gratings, we have designed fiber grating multi-dimensional strain, displacement, temperature, pressure, and vibration sensing probes. Minimizing the size of the sensor involves the sensitivity increase/decrease and structure design of multi-dimensional and multi-parameter fiber grating sensing probes.

(2) Wavelength demodulation technique of fiber gratings for sensing applications

In order to ensure the low power consumption level, this work is focused on the research on the wavelength demodulation technique of miniaturized fiber gratings for applications in the WSN, including the studies on the module design, compatibility, minimization, high stability, and reliability.

(3) Data acquisition and communication technique

In this section, based on the advanced reduced instruction set computer (RISC) machines (ARM), DSP and field-programmable gate array (FPGA) design, we set up the hardware development platform for the fiber-grating-based wireless sensing unit to achieve the wavelength demodulation of fiber gratings, data fusion of status parameters, data storage and wireless radiation driving.

(4) Power supply and low-power design

Considering the inherent functionality of the wireless sensor networks, the sensor node should be battery-powered. Thus, the primary aim of this work is to minimize the power system for a longer lifetime of individual battery components.

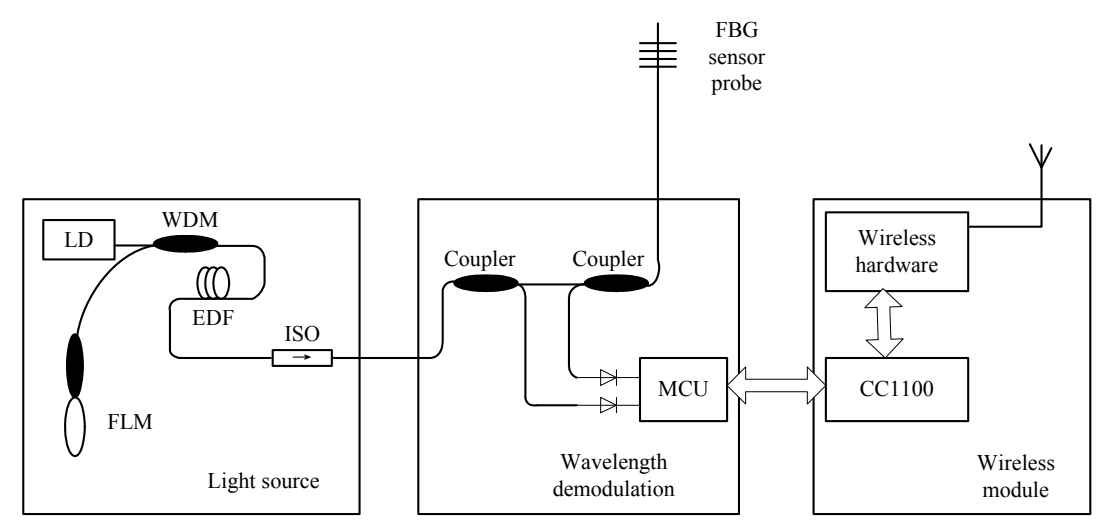

Fig. 2 Principle block diagram inside a sensor node.

Figure 3 shows the photograph of a sensor node in the fiber-grating-based WSNs.
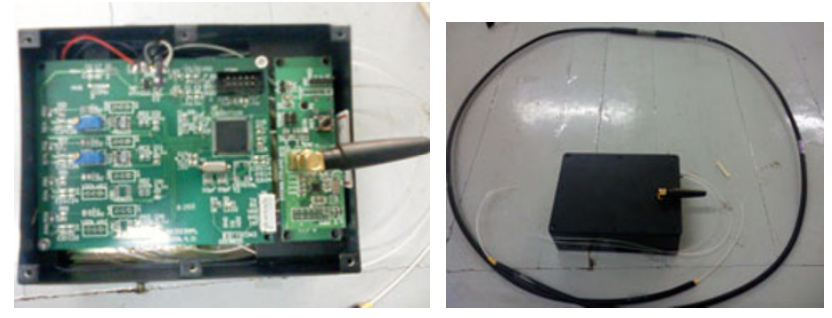

Fig. 3 Photograph of a sensor node.

\subsection{Wireless communication technique}

The distributed measurement information could be acquired from the sensor nodes to wirelessly form the sensing network and gather the information to control the terminal via the optimal approaches including the wireless communication technique, networking technique and routing algorithm.

(1) Wireless communication technique

Wireless communication technique generally 
refers to the frequency methods, power methods and encoding methods on the hardware. The data communication with a $433-\mathrm{MHz}$ carrier wave was adopted in our scheme, and the maximum communication radius was more than 100 meters.

(2) Routing algorithm

In order to effectively solve the several problems in WSN applications such as the large scale networking, dynamic adaptation of sensors and limited transmission distance, we adopted the low energy adaptive clustering hierarchy (LEACH) algorithm, the principle block diagram of which is illustrated as below:

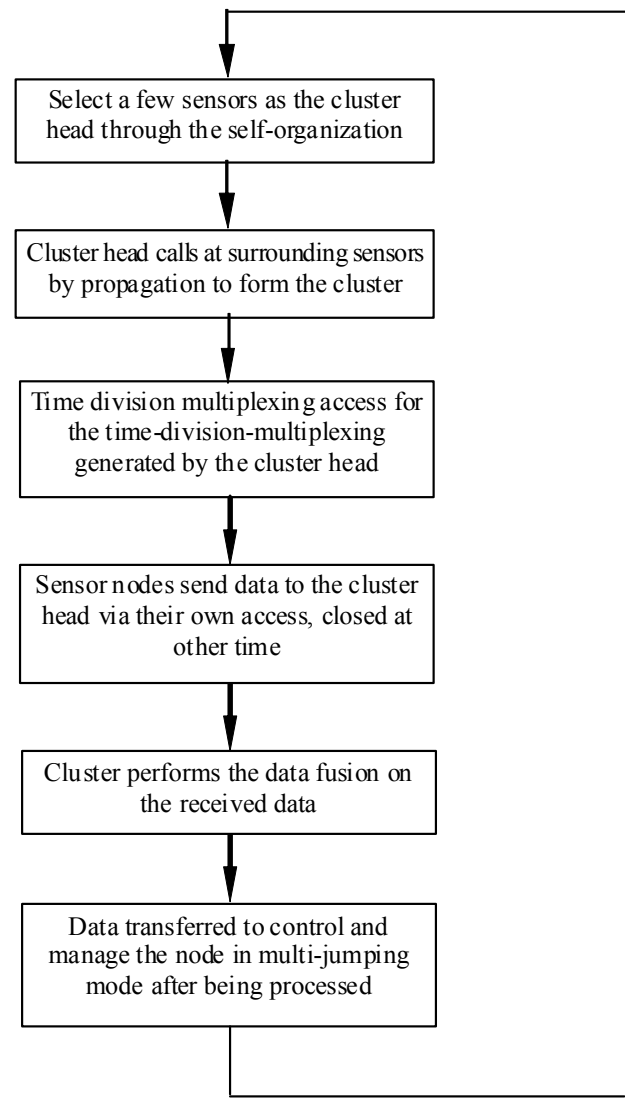

According to the characteristics of fiber-grating-based WSNs, the LEACH protocol was selected as the routing protocol of our system. The LEACH protocol runs by rounds, and one round could be divided into two stages: setup stage and steady stage. In the setup stage, a few sensor nodes are randomly selected as the cluster head, namely the sink node, via self-organization. Then the cluster head will call at common sensor nodes in charge of the formation of the cluster with the closest cluster head according to the received signal intensity. When all of the sensor nodes have joined a certain group, every sensor node will inform its cluster head selection result through the carrier sense multiple access (CSMA) physical protocol. Accordingly, the cluster head will generate a time division multiplexing access (TDMA) and propagate the TDMA to all of the group member sensors. Thus, every sensor node knows which access it belongs to and starts to send the data to the cluster head when its own access is activated.

The transceiver at every sensor node is only turned on when its own access is activated and is closed at other time to economize the energy consumption. In contrast, the transceiver at the cluster head is always in operation to receive the data from the respective group member sensor nodes, perform data processing and report the processing result to the distant base station (reception node). As one round is over, the group structure will be re-organized, the cluster head will be re-elected, and the group structure will be re-established. In other words, the above round is repeated.

The communication between the cluster head and management node is conducted in the multi-jumping mode, as shown in Fig. 4.

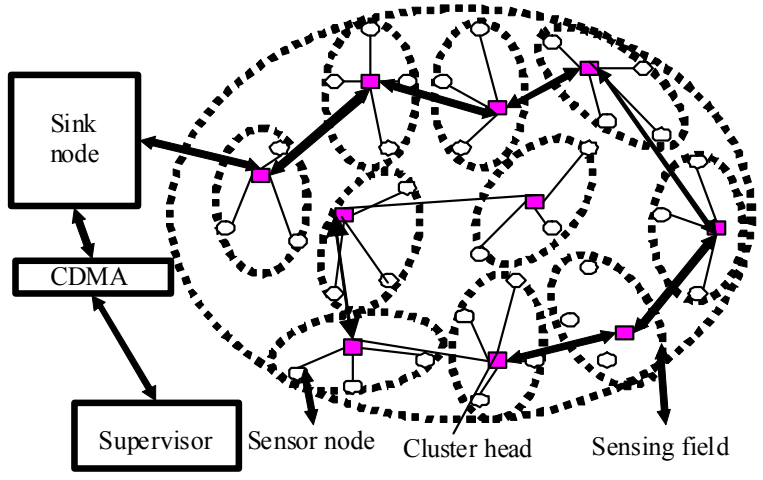

Fig. 4 Route topology map of fiber-grating-based WSNs.

Finally, we have achieved a 20-node fiber-grating-based wireless sensor network for the demonstration. Each sensor node is integrated with the light source, 1-3 FBG sensing probes, 
wavelength demodulation and wireless communication module. The sensing probe is designed to be sensitive to temperature, strain, pressure and vibration, etc. These sensor nodes can exchange sensing data with the control center by self-organized clusters and routing protocols. And the maximum communication radius of a sensor node is over $170 \mathrm{~m}$. The sensor node is battery-powered with a survival lifetime of up to 120 days at the network refresh rate of 5 minutes.

\section{Summary and prospect}

Due to their distinguished advantages such as immunity to the electro-magnetic perturbation, capability of the long-distance remote sensing, ease of the networking, and applicability for applications in severe environments, fiber sensors are technologically complimentary with electrical sensors. Compared with electrical sensors, it is more convenient to utilize fiber sensors to construct large capacity networks, which is an outstanding merit of the fiber grating senor network. In the future, fiber sensor networks would be developing toward digitalization, intelligence and compactness, and the so called optical fiber sensor Internet (OFS Internet) will finally be realized by collaboration with other communication networks. By combining the advanced fiber grating sensing technique with the wireless sensing network technique, our work is an attempt on the large scale fiber grating sensor networking. A 20-node fiber-grating-based wireless sensing network has been achieved. Based on the same method, the network capacity can be easily expanded. However, the power supply method and demodulation method of fiber grating sensors turn to be the threshold obstacles for the further development of the fiber-grating-based WSN.

\section{Acknowledgement}

Our work is supported by the 863 National High Technology Program of China under Grant No 2006AA01Z217 "Study on multi-dimensions multi-parameters fiber Bragg grating wireless sensor networks".

Open Access This article is distributed under the terms of the Creative Commons Attribution License which permits any use, distribution, and reproduction in any medium, provided the original author(s) and source are credited.

\section{References}

[1] B. Liu, J. Zeng, C. X. Xiao, et al., "A novel method for the demodulation of fiber Bragg grating sensing system based on chirp grating," in Proc. SPIE, vol. 5279, pp. 555-559, 2004.

[2] L. F. Xue, Q. D. Zhao, J. G. Liu, G. L. Huang, T. Guo, and X. Y. Dong, "Force sensing with temperature self-compensated based on a loop thin-wall section beam," IEEE Photonics Technology Letters, vol. 18, no. 1, pp. 271-273, 2006.

[3] W. G. Zhang, Y. L. Huang, Xiang Yang, T. Z. Rong, Q. D. Zhao, G. Y. Kai, S. Z. Yuan, and X. Y. Dong, "Temperature-independent stress and displacement bi-directional sensing tuned by applying bilateral cantilever beam," Chinese Physical Letters, vol. 19, no. 1, pp. 76-78, 2002 (in Chinese).

[4] W. G. Zhang, X. Y. Dong, D. J. Feng, Z. X. Qin, and Q. D. Zhao, "Linear fiber-grating-type sensing tuned by applying torsion stress," Electronics Letters, vol. 36, no. 20, pp. 1686-1688, 2000.

[5] T. Guo, Q. D. Zhao, H. Zhang, C. S. Zhang, G. L. Huang, L. F. Xue, and X. Y. Dong, "Temperature-insensitive fiber Bragg grating dynamic pressure sensing system," Optics Letters, vol. 31, no. 15, pp. 2269-2271, 2006.

[6]P. M. Cavaleiro, F. M. Araujo, and A. B. Lobo Ribeiro, "Metal-coated fiber Bragg grating sensor for electric current metering," Electronics Letters, vol. 34, no. 11, pp. 1133-1135, 1998.

[7] X. Y. Dong, H. Y. Meng, G. Y. Kai, Z. G. Liu, and X. Y. Dong, "Bend measurement with chirp of fiber Bragg grating," Smart Materials and Structures, vol. 10, no. 5, pp. 1111-1113, 2001.

[8] X. Y. Dong, Y. Q. Liu, Z. G. Liu, and X. Y. Dong, "Simultaneous displacement and temperature measurement with cantilever-based fiber Bragg grating sensor," Opt. Comm., vol. 192, no. 1-3, pp. 213-217, 2001.

[9] W. G. Zhang, Z. W. Xu, X. P. Yang, G. Y. Kai, S. Z. Yuan, and X. Y. Dong, "Study of fiber-type sensor of refractive indices and concentration of liquids," in Proc. SPIE, vol. 4595, pp. 209-212, 2001.

[10] C. Y. Lin, L. A. Wang, and G. W. Chern, 
"Corrugated long-period fiber grating as strain, torsion, and bending sensors," Journal of Lightwave Technology, vol. 19, no. 8, pp. 1159-1168, 2001.

[11] A. D. Kersey, "Optical fiber sensors for permanent downwell monitoring applications in the oil and gas industry," IEICE Trans. Electron., vol. E83-C, no. 3, pp. 400-404, 2000.

[12] R. Arai, A. Sumita, and S. Makino, "Large-scale hybrid monitoring system for temperature, strain, and vibration using fiber Bragg grating sensors," in Proc. SPIE, vol. 4920, pp. 62-72, 2002.

[13] W. W. Morey, G. Meltz, and W. H. Glenn, "Fiber Bragg grating sensors," in Proc. SPIE (Fiber Optics \& Laser Sensors VII), vol. 1169, pp. 98-107, 1989.

[14] L. Zhang, Y. Liu, J. A. R. Williams, and I. Bennion, "Enhanced FBG strain sensing multiplexing capacity using combination of intensity and wavelength dual-coding technique," Photonics Technology Letters, vol. 11, no. 12, pp. 1638-1640, 1999.

[15] P. C. Peng, et al., "Intensity and wavelength-division multiplexing FBG sensor system using a tunable multiport fiber ring laser," Photonics Technology Letters, vol. 16, no. 1, pp. 230-232, 2004.

[16] J. M. Baptista, S. Abad, G. M. Rego, et al., "Wavelength multiplexing of frequency-based self-referenced fiber optic intensity sensors," Optical Engineers, vol. 43, no. 3, pp. 702-707, 2004.

[17] Y. L. Yu, H. Y. Tan, et al., "Active spatial wavelength division multiplexed fiber Bragg grating sensor system," Chinese Jounal of Lasers, vol. 29, no. 2, pp. 131-134, 2002 (in Chinese).

[18] Y. L. Yu, H. Y. Tam, et al., "Passive fiber Bragg grating sensor system using combined spatial and time division multiplexing technique," Acta Optica Sinica, vol. 21, no. 11, pp. 1313-1315, 2001 (in Chinese).

[19] L. B. Yuan and J. Yang, "Two-loop-based low-coherence multiplexing fiber-optic sensor network with a Michelson optical path demodulator," Optics Letters, vol. 30, no. 6, pp. 601-603, 2005.

[20] C. Y. Chong and S. P. Kumar, "Sensor networks: evolution, opportunities, and challenges," in Proc. IEEE, vol. 91, no. 8, pp. 1247-1256, 2003.

[21] K. Chintalapudi, et al., "Monitoring civil structures with a wireless sensor network," IEEE Internet Computing, vol. 10, no. 2, pp. 26-34, 2006.
[22] J. S. Lee and P. L. Hsu, "Design and implementation of the SNMP agents for remote monitoring and control via UML and Petri Nets," IEEE Transactions on Control Systems Technology, vol. 12, no. 2, pp. 293-302, 2004.

[23] J. L. Peterson, Petri Net Theory and Modeling of Systems, New Jersey: Prentice Hall, 1981, pp. 280.

[24] E. Waltz and J. Lilnas, Multisensor data fusion. Boston: Artech House, 2000.

[25] D. L. Hall and J. Llinas, "An introduction to multisensor data fusion," in Proc. IEEE, vol. 85, no. 1, pp. 6-23, 1997.

[26] B. Dasarathy, "Information fusion, data mining and knowledge discovery," Information Fusion, vol. 4, no. 1, pp. 1-3, 2003.

[27] J. K. Uhlmann, "Covariance consistency method for fault-tolerant distributed data fusion," Information Fusion, vol. 4, no. 3, pp. 201-215, 2003.

[28] G. Saporta, "Data fusion and data grafting," Computational Statistics and Data Analysis, vol. 38, no. 4, pp. 465-473, 2002.

[29] H. Z. Sasiadek, "Sensor fusion," Annual Reviews in Control, vol. 26, no. 26, pp. 203-228, 2002.

[30] T. Richard, "Principles of effective multi-sensor data fusion," Military Technology, vol. 27, no. 5, pp. 29-37, 2003.

[31] H. D. Whyte, "Sensor models and multi-sensor integration," The International Journal of Robotics Research, vol. 7, no. 6, pp. 97-113, 1988.

[32] D. Y. Yang and Y. Yamane, "Multi-sensor data fusion and its application to industrial control," in Proc. of the 39th SICE Annual Conference International Session Papers (2000), vol. 3, pp. 215-220, 2002.

[33] V. Megalooikonomou and Y. Yesha, "Space efficient quantization for distributed estimation by a multi-sensor fusion system," Information Fusion, vol. 5, no. 4, pp. 299-308, 2004.

[34] X. H. Huang, M. Wang, and Z. Y. Huang, "Information acquisition and data fusion structures for multi-sensor systems," Science and Technology of Information Acquisition and their Applications, vol. 8, no. 11, pp. 22-26, 2003.

[35] G. Werner-Allen, et al., "Deploying a wireless sensor network on an active volcano," IEEE Internet Computing, vol. 10, no. 2, pp. 18-25, 2006. 